\title{
The Effects of Heat Shock Proteins on Delivery of HIV-1 Nef Antigen in Mammalian Cells
}

\author{
Alireza Milani $^{1}{ }^{(\mathbb{D}}$, Fatemeh Rouhollah $^{1}$, Maryam Naseroleslami $^{1}{ }^{\circledR}$, Azam Bolhassani $^{2 *}(\mathbb{D}$ \\ ${ }^{1}$ Department of Cellular and Molecular Biology, Faculty of Advanced Sciences and Technology, Tehran Medical Sciences, \\ Islamic Azad University, Tehran, Iran; ${ }^{2}$ Department of Hepatitis and AIDS, Pasteur Institute of Iran, Tehran, Iran
}

\begin{abstract}
A R T ICLE IN F O
A B S T R A C T

\section{Original Article}

VacRes, 2020

Vol. 7, No. 1, 54-59

Received: August 23, 2020

Accepted: September 23, 2020

Pasteur Institute of Iran

*Corresponding Author:

Azam Bolhassani, PhD, Department of

Hepatitis and AIDS, Pasteur Institute

of Iran, Tehran, Iran.

Email: azam.bolhassani@yahoo.com

Tel/Fax: (+98)2166953311 Ext. 2240

$/(+98) 2166465132$

KEYWORDS: HIV-1, Nef Antigen, Heat Shock Protein, Hsp27, Hsp70, DNA Vaccine

Introduction: Vaccine design is mainly considered as a therapeutic strategy to elicit HIVspecific immunity. DNA vaccines encoding an antigen and also an adjuvant can induce an effective adaptive immunity. Due to having numerous roles in viral infection, the HIV-1 Nef protein is considered as an antigen candidate for development of therapeutic vaccines. A variety of adjuvants and delivery systems have been utilized to increase the potency of DNA vaccines against viral infections, such as heat shock proteins (HSPs) which possess chaperon activity and immunostimulatory properties. Methods: pEGFP mammalian expression vectors harboring nef, hsp27, hsp27-nef, hsp70 and hsp70-nef genes were prepared in large scale. Their concentration and purity were assessed by NanoDrop spectrophotometry. Human embryonic kidney 293T cells (HEK-293T) were grown in DMEM culture medium and transfected with these constructs using Lipofectamine 2000 transfection reagent. After 48 hours, their transfection efficiency was evaluated using fluorescent microscopy and flow cytometry. Results: The pEGFP-nef, - $h s p 27,-h s p 27-n e f$, $-h s p 70$ and $-h s p 70-n e f$ constructs were successfully prepared in large scale and high purity. The results of cell transfection with each construct showed that the percentages of Nef-GFP, Hsp27-GFP, Hsp27-Nef-GFP, Hsp70-GFP, Hsp70-Nef-GFP, Hsp70-GFP + Nef-GFP and GFP expression were 53.1 \pm 0.2, 64.22 \pm 0.8, 57.1 \pm 0.7, 68.8 $\pm 1.0,61.7 \pm$ 0.7, $77.4 \pm 1.5$ and $81.8 \pm 1.8$, respectively. Conclusion: These data showed that the potency of Hsp70 was more than Hsp27 for increasing Nef-GFP expression in HEK-293T cells. Moreover, the delivery of pEGFP-nef along with pEGFP-hsp70 increased the rate of GFP population in the cells as compared to that in pEGFP-hsp70-nef. These constructs will be used for development of HIV-1 DNA vaccine in animal model in near Future.
\end{abstract}

\section{Citation:}

Milani A, Rouhollah F, Naseroleslami M, Bolhassani A. The Effects of Heat Shock Proteins on Delivery of HIV-1 Nef Antigen in Mammalian Cells. vacres. 2020; 7 (1) :54-59. DOI: 10.29252/vacres.7.1.54

\section{INTRODUCTION}

Designing an effective vaccine against human immunodeficiency virus (HIV) has remained as an intractable challenge [1-3]. Some evidences confirmed the importance of virus-specific cellular immune responses in controlling HIV-1 replication; thus different therapeutic vaccine strategies based on viral or bacterial vectors, DNA, RNA, peptide and protein are being developed in preclinical or clinical trials [4]. It has been determined that DNA vaccination can effectively stimulate both innate and adaptive immunities (e.g. CD8+ T cell and CD4+ T helper cell immune responses) [5]. The plasmid DNA encoding a therapeutic antigen can be directly designed based on pathogen sequences, allowing flexibility and speed in preclinical testing and subsequently rapid transition to clinical scale up [6]. Among the HIV-1 genes, the nef regulatory gene plays a critical role in viral infection including down-regulation of cell surface receptors such as MHC-I and $\mathrm{CD} 4$, enhancement of viral replication, and alteration of $\mathrm{T}$ cell activation. Also, Nef can be considered as an attractive antigen candidate in therapeutic vaccine development due to its multiple immunogenic epitopes [7-9)]. Practical considerations of DNA vaccines as an alternative to other therapeutic methods against HIV depends on the improvement of delivery systems and also adjuvants. The endogenous adjuvants such as heat shock proteins (HSPs) has been suggested to induce antigenspecific humoral and cellular immunity, effectively [10]. Among HSPs, small heat shock protein 27 (Hsp27) can modulate the immune system through interaction with toll like receptors-2 and -4 [11]. Hsp70 could increase the presentation and cross-presentation of antigens to the immune system for development of innovative therapeutic vaccines against cancers 
and chronic viral infections [12]. To develope DNA vaccine, in vitro expression of the gene constructs could facilitate rapid screening and selection of potential vaccine candidates. Herein, we prepared DNA constructs of Nef, Hsp27, Hsp27-Nef, Hsp70 and Hsp70-Nef and evaluated their expression in a mammalian cell line. These constructs were transfected to the HEK-293T cell line using Lipofectamine 2000 transfection reagents and their expression was assessed by fluorescent microscopy and flow cytometry.

\section{MATERIALS and METHODS}

\section{Preparation of the Recombinant Plasmids}

To monitor the efficiency of transfection, pEGFP-N1 and pEGFP-N3 eukaryotic vectors encoding enhanced green fluorescent protein (EGFP) were employed in this study. The constructions of the full length pEGFP-nef, pEGFP-hsp27 and pEGFP- $h s p 27-n e f$ genes were described in our previous study [13]. Moreover, the full length of $h s p 70$ gene was prepared in a pUC57 cloning vector by Biomatik Company (Canada). To generate pEGFP-hsp70, the fragment was subcloned into pEGFP-N3 eukaryotic expression vector in NheV/ SalI sites (Fermentas) (Fig. 1.A). Also, to make pEGFP-hsp70-nef, the nef fragment was amplified by PCR from pUC19-nef using primers designed to generate EcoRI and SalI restriction sites at 5' and 3' ends of the amplified fragment, as shown below. Then, the nef gene was ligated in a linearized pEGFP-hsp70 between EcoRI and SalI sites using T4 DNA Ligase (Fermentas) (Fig. 1.B).

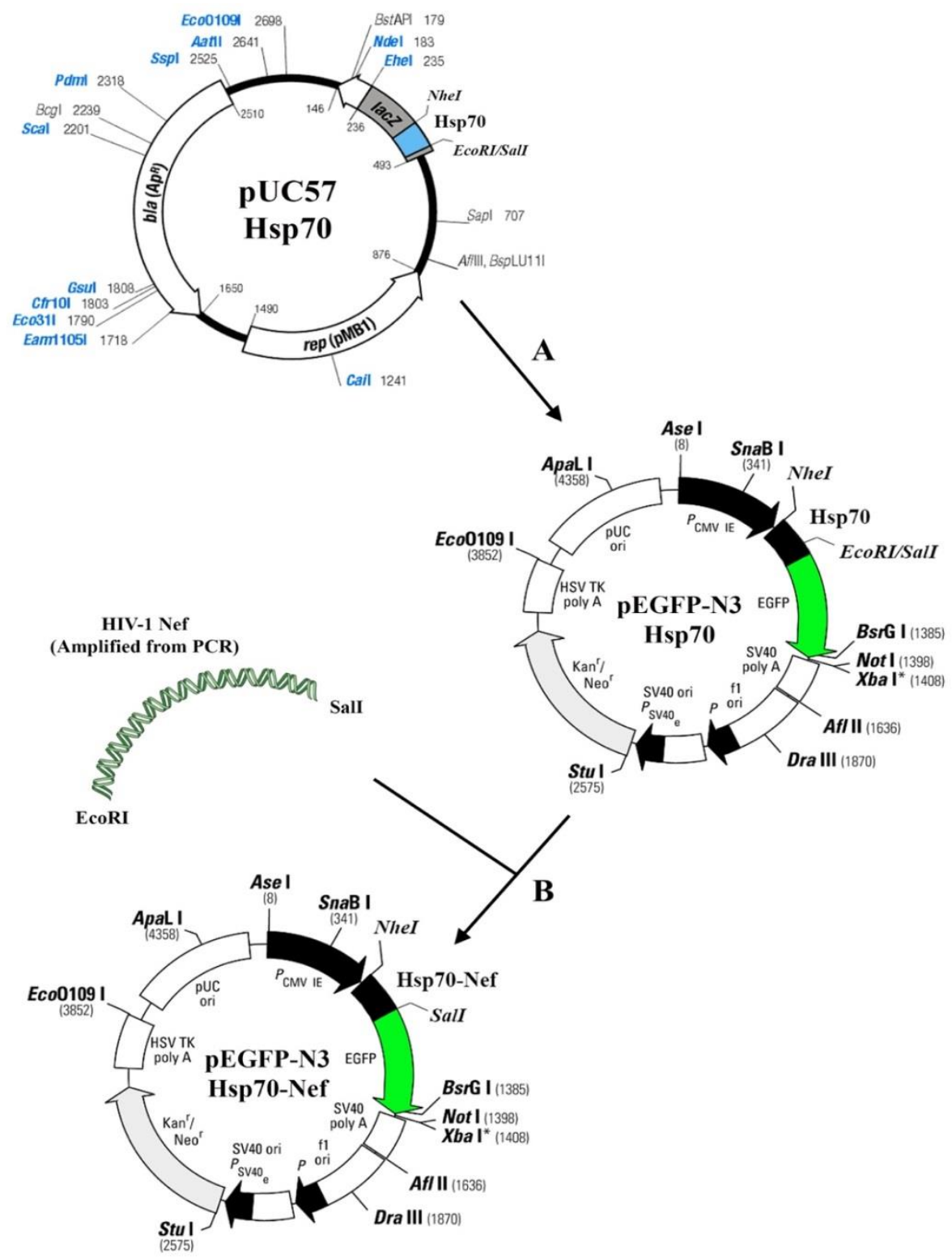

Fig. 1. Schematic diagram for the construction of pEGFP- $h s p 70$ and pEGFP- $h s p 70-n e f$. (A) pEGFP- $h s p 70$ : The $h s p 70$ fragment was digested from pUC57-hsp70 with NheI/SalI and subcloned into the pEGFP-N3 eukaryotic vector. (B) pEGFPhsp70-nef: The PCR product of nef gene was digested with EcoRI/SalI and cloned into the recombinant pEGFP-Hsp70 vector. 
After transformation of E.coli $\mathrm{DH} 5 \alpha$ strain with the ligated solution, the recombinant plasmids were purified from clones using plasmid DNA extraction mini-kit (FavorPrep ${ }^{\mathrm{TM}}$, Taiwan) according to manufacturer's instructions. The concentration and purity of plasmid DNA were determined by NanoDrop ${ }^{\mathrm{TM}}$ Lite Spectrophotometer (Thermo Fisher Scientific).

\section{Cell Culture}

Human Embryonic Kidney 293T (HEK-293T) cell line (Pasteur institute of Iran) were grown in Dulbecco's Modified Eagle's Medium (DMEM; Sigma), supplemented with 10\% fetal bovine serum (FBS, Gibco), pen/strep (100U/ml penicillin and $0.1 \mathrm{mg}$ Streptomycin; Gibco). The cells were seeded at $5 \times 10^{4}$ cells/well in 24 -well plates, approximately 24 hours prior to transfection and incubated under standard cell culture conditions $\left(37^{\circ} \mathrm{C}, 5 \% \quad \mathrm{CO}_{2}\right.$, humidified air) for achieving approximately $80 \%$ confluency.

\section{Transfection}

The plasmid DNAs, namely pEGFP-N1, pEGFP-nef, pEGFP- $h s p 27$, pEGFP- $h s p 27-n e f$, pEGFP- $h s p 70$ and pEGFPhsp70-nef were transfected using Lipofectamine ${ }^{\mathrm{TM}} 2000$ (Invitrogen, USA) based on the manufacturer's protocols. Also, the cells were cotransfected with pEGFP-nef + pEGFP-hsp70 for comparison with transfection of pEGFP-hsp70-nef construct. For production of lipofectamine/ plasmid DNA complex, $50 \mu \mathrm{l}$ of serum-free DMEM was mixed with $2 \mu \mathrm{l}$ of lipofectamine and incubated for $5 \mathrm{~min}$ in room temperature. Then, $50 \mu \mathrm{l}$ incomplete DMEM was mixed with $1 \mu \mathrm{g}$ of each plasmid DNA and added to lipofectamine solution. The mixtures were incubated for $30 \mathrm{~min}$ in room temperature to form the DNA/lipofectamine complexes. The complexes were then added to each well and the medium was replaced after $5 \mathrm{~h}$ with pre-warmed complete DMEM (5\% FBS). Finally, the plates were incubated for 48 hours at $37^{\circ} \mathrm{C}$ for measuring transient transfection efficiency. The cells transfected with pEGFP-N1 vector were used as a positive control. The untreated cells were considered as a negative control.

\section{In Vitro Transfection Assay}

Fluorescent microscopy and flow cytometry were used for monitoring the efficiency of plasmid DNA uptake into the cells using GFP reporter. The expressions of Nef-GFP, Hsp27-GFP, Hsp27-Nef-GFP, Hsp70-GFP, Hsp70-Nef-GFP and GFP proteins were detected by fluorescent microscopy (Envert Fluorescent Ceti, Korea) and quantified by a flow cytometer (Partec, Germany) after 48 hours cell transfection. For flow cytometry analysis, the cells were harvested by trypsin $1 \mathrm{X}$ and the cell pellets were resuspended in PBS $1 \mathrm{X}(\mathrm{pH}$ 7.4). The expressions of the fluorescent genes were measured in FL1 channel using an excitation filter $(485 \mathrm{~nm})$ and an emission filter $(535 \mathrm{~nm})$.

\section{Statistical Analysis}

Prism 8.3 software (GraphPad, San Diego, California, USA) was used for statistical analysis. The differences between the control and the test groups were assessed using one-way ANOVA (GraphPad Prism, GraphPad Software) where $\mathrm{p}<0.05$ was considered statistically significant.

\section{RESULTS}

\section{Generation of the Recombinant DNA Constructs}

The subcloning of $h s p 70$ gene into pEGFP-N3 vector was confirmed by the presence a $\sim 1950 \mathrm{bp}$ fragment on $1 \%$ gel electrophoresis. Moreover, a clear band of $\sim 2600 \mathrm{bp}$ for the hsp70-nef fusion was observed on the gel after NheI/SalI double-digestion as shown in Fig. 2.

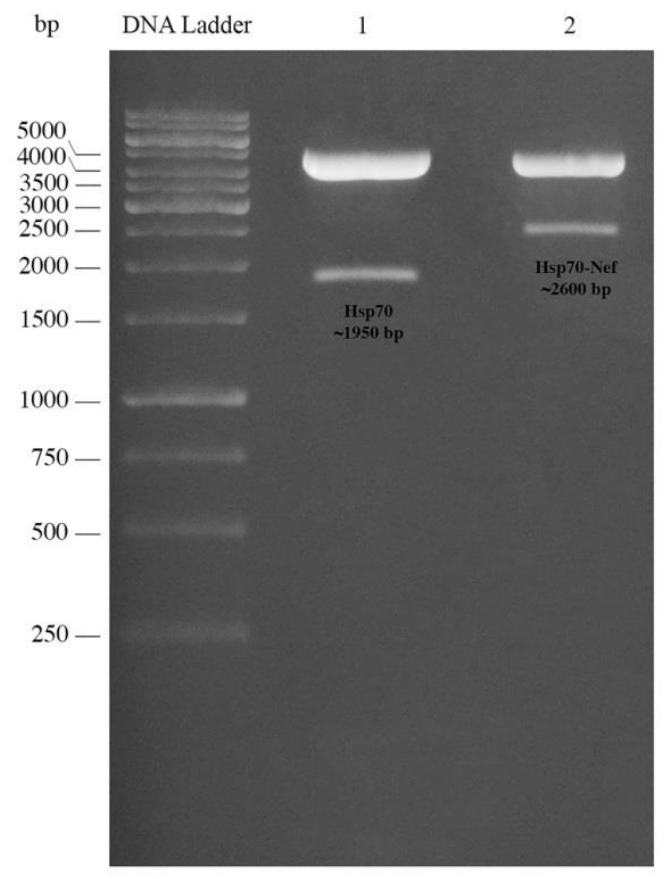

Fig. 2. Confirmation of the recombinant plasmids by double-digestion on gel electrophoresis.

Lanes 1 and 2 represent the double digested products using NheI/SalI restriction enzymes for pEGFP- $h s p 70(\sim 1950 \mathrm{bp})$ and pEGFP-hsp70-nef ( $2600 \mathrm{bp})$, respectively.

The nef, hsp27 and hsp27-nef genes were previously confirmed as clear bands of $\sim 648 \mathrm{bp}, \sim 720 \mathrm{bp}$ and $\sim 1368 \mathrm{bp}$ on agarose gel [13]. The pEGFP-nef, pEGFP-hsp 27 and pEGFP$h s p 27-n e f$, pEGFP-hsp70 and pEGFP-hsp70-nef were prepared with high purity.

\section{Evaluation of Transfection Efficiency Using Fluorescent Microscopy and Flow Cytometry}

The DNA transfection efficiency using Lipofectamine was detected by fluorescent microscopy and flow cytometry. These results were determined by the percentage of protein expression using GFP reporter. The percentage of Nef-GFP and GFP expression were $53.1 \pm 0.2$ and $81.8 \pm 1.8$, respectively (Fig. 3).

The transfection rates of Hsp27-encoding DNA constructs were $64.22 \pm 0.8$ and $57.1 \pm 0.7$ for Hsp27-GFP and Hsp27Nef-GFP, respectively. Also, the transfection percentages of Hsp70-encoding DNA constructs were $68.8 \pm 1.0,61.7 \pm 0.7$ and $77.4 \pm 1.5$ for Hsp70-GFP, Hsp70-Nef-GFP and Hsp70GFP + Nef-GFP, respectively. The transfection efficiency of Hsp-encoding constructs was shown in Fig. 4. 


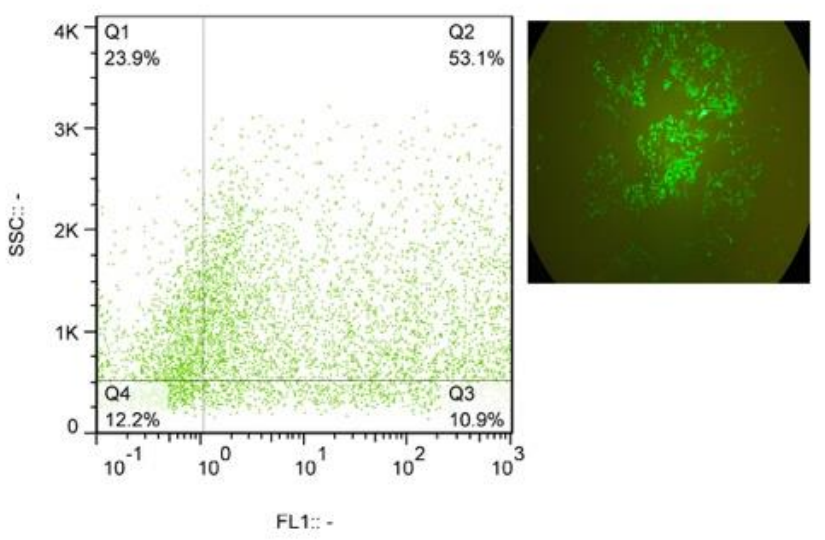

Nef-GFP

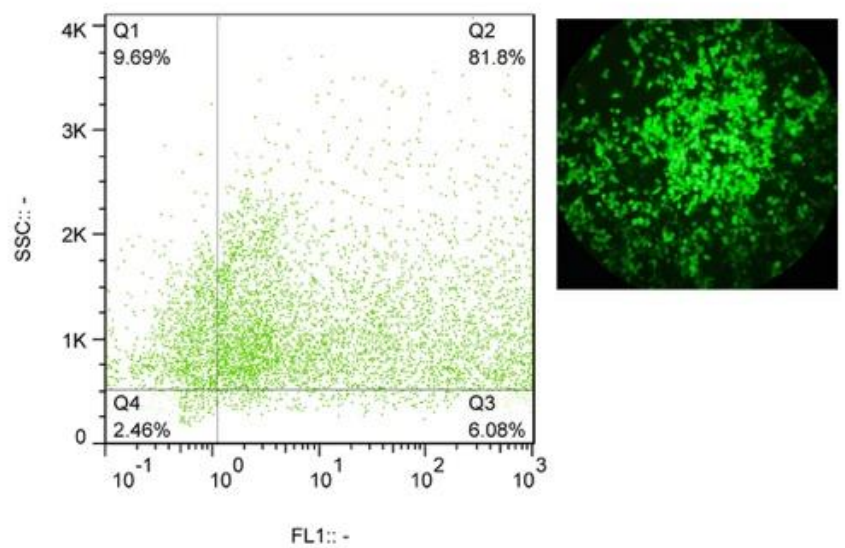

GFP

Fig.3. Analysis of Nef-GFP and GFP expression in HEK-293T cells by Lipofectamine 2000 transfection reagent using fluorescent microscopy and flow cytometry. The pEGFP-N1 was used as a positive control. The transfection rates of Nef-GFP and GFP expression were $53.1 \pm 0.2$ and $81.8 \pm 1.8$, respectively.

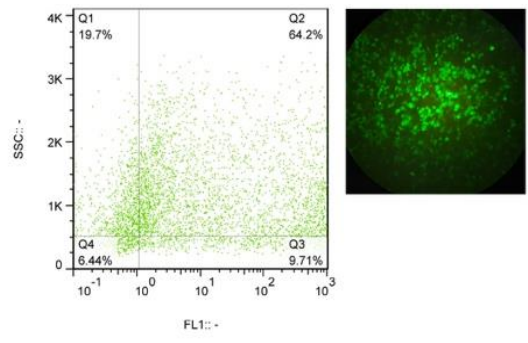

Hsp27-GFP

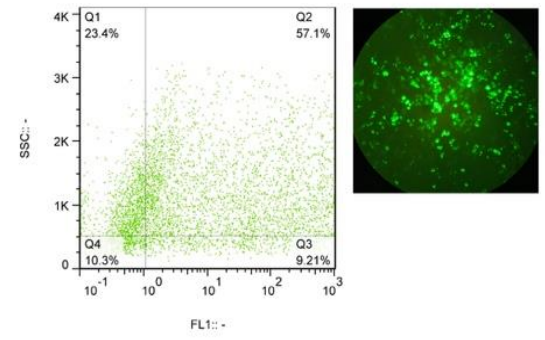

Hsp27-Nef-GFP

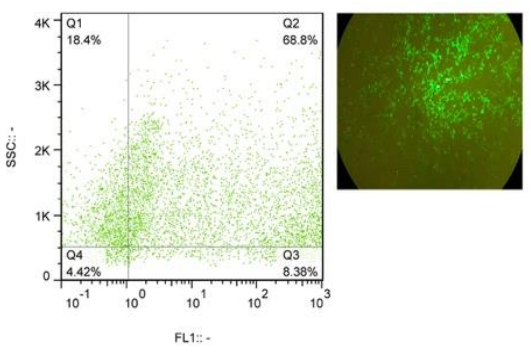

Hsp70-GFP

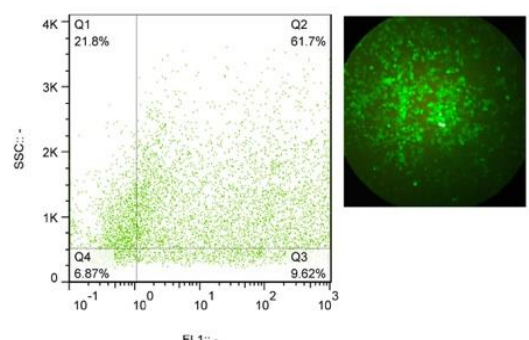

Hsp70-Nef-GFP

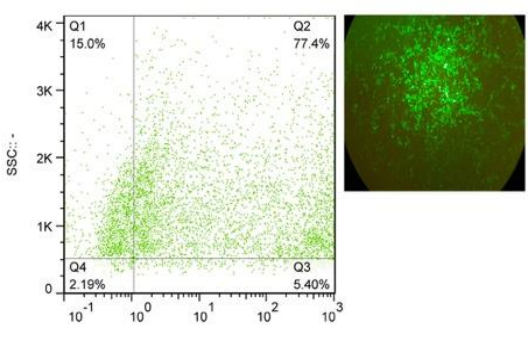

FL1:-

Hsp70-GFP + Nef-GFP

Fig. 4. Analysis of gene expression in HEK-293T cells by Lipofectamine 2000 transfection reagent using fluorescent microscopy and flow cytometry. The transfection rates of Hsp27-encoding DNA constructs were $64.22 \pm 0.8$ and $57.1 \pm 0.7$ for Hsp27-GFP and Hsp27-Nef-GFP, respectively. Also, the transfection percentages of Hsp70-encoding DNA constructs were $68.8 \pm 1.0,61.7 \pm 0.7$ and $77.4 \pm 1.5$ for Hsp70-GFP, Hsp70-Nef-GFP and Hsp70-GFP + Nef-GFP, respectively. 
Flow cytometry analysis indicated that the cell penetration of pEGFP-hsp70-nef using Lipofectamine transfection reagent was more than pEGFP-hsp27-nef ( $\mathrm{p}<0.05)$. Also, the expression of Hsp70-GFP was more than Hsp27-GFP ( $p<$ $0.05)$; thus Hsp70 could increase Nef-GFP expression in the cells, significantly higher than Hsp27 ( $\mathrm{p}<0.05)$. Also, the delivery of pEGFP-nef along with pEGFP- $h s p 70$ increased the rate of GFP population in the cells indicating high transfection efficiency using lipofectamine in HEK-293T cells. The transfection efficiency is summarized in Fig. 5.

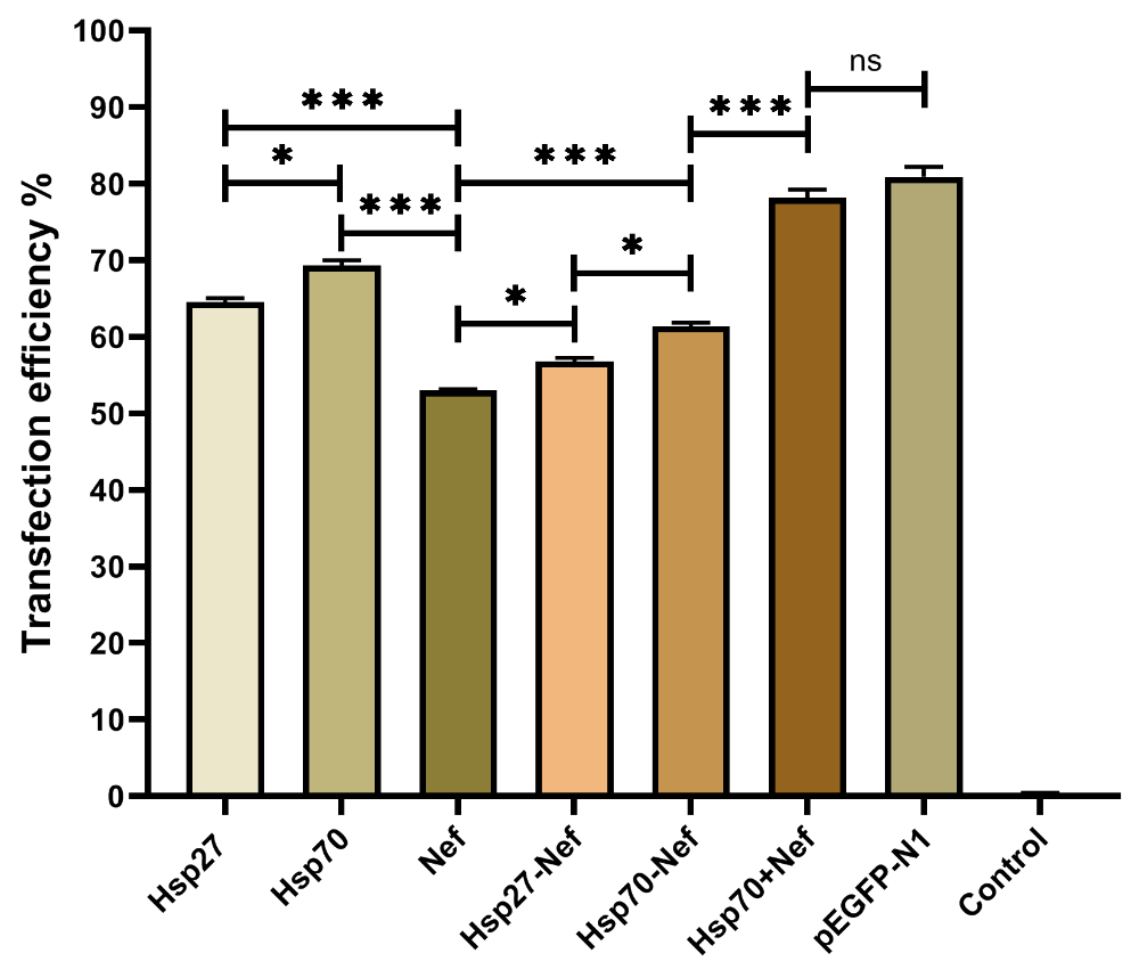

Fig. 5. Comparison of Hsp27 and Hsp70 in delivery of Nef antigen into HEK-293T cells. The expression of the Hsp70-Nef fusion protein was significantly higher than the Hsp27-Nef fusion protein $(\mathrm{p}<0.05)$. $(* \mathrm{p}<0.05$, *** $\mathrm{p}<0.001$, ns: non-significant).

\section{DISCUSSION}

DNA vaccines were broadly utilized in animals; however, they are slightly applied in clinical studies because of their low immunogenicity. Recently, several approaches have been evaluated to improve the potency of DNA vaccines such as advancement in design of the DNA vector, the use of adjuvants and the delivery systems [14]. The use of liposome as a carrier has become a popular DNA delivery method in vitro. Plasmid DNA can be bound to the liposome which facilitates its delivery into the cells. In one study, transfection of enhanced green fluorescent protein (EGFP) using Lipofectamine 3000 has shown strong expression of EGFP in HEK293 cells, mouse primary cortical neurons and human umbilical vein endothelial cells (HUVECs). Also, Cx43 shRNA lentivirus combined with Lipofectamine 3000 transfection reagent has been shown to achieve $90 \%$ Cx43 knockdown efficacy in HUVECs [15]. In another study, Zhou et al. have shown that the expression of sag4 gene cloned in pEGFP-C1 eukaryotic vector in HEK 293$\mathrm{T}$ cells, using Lipofectamine 2000 for development of a DNA vaccine against $T$. gondii infection [16].

Recombinant DNA technology allows the expression of antigens in their native conformation and thereby induction of cellular immunity against pathogens such as HIV-1. Based on previous studies, HIV-1 Nef has been known as a promising antigen candidate for HIV-1 vaccine development due to conservation and immunogenicity as well as harboring several T- and B-cell epitopes [7, 17, 18]. For evaluating the delivery of Nef from $\mathrm{T}$ cells to endothelial cells, the Jurkat cells have been transfected with HIV-1 Nef, using Lipofectamine LTX reagent and after 48 hours post transfection, these Jurkat cells were cocultured with HCAEC cells. The reported data have demonstrated that the efficiency of Nef transfection was $20 \%$ in Jurkat cells, and could be transferred from Jurkat to endothelial cells, indicating direct cell-to-cell contact. Moreover, the efficiency of Nef transfection in HCAEC endothelial cells has been estimated as $\sim 70 \%$ using flow cytometry [19]. In the recent study, the efficiency of HIV-1 Nef expression in HEK-293T using Lipofectamine was $~ 53 \%$. These differences may be due to the fact that the transfection efficiency of chemical methods is dependent on the DNA vector, the reagents and the cell type.

Yamaoka et al. have reported a novel DNA vaccine design based on Hsp70 and Ovalbumin (OVA) MHC class I epitope peptide. Before mice immunization with the Hsp70-CTL epitope fusion construct, this construct was transfected in COS7 cells using Lipofectamine 2000 and its expression was confirmed by Western blotting [20]. Another study has also represented transfection of human papillomavirus (HPV)-16 E7-Hsp27 fusion in COS-7 cells using lipofectamine 2000 for screening potential expression of DNA vaccine candidate in eukaryotic cells [21]. To investigate the heat-shock effect on gene expression, a pGL3-Hsp70 has been transfected into B16 cells, using Lipofectamine LTX reagent at a ratio of $4: 1$ reagent/DNA. After 24 hours, the cells were heated at $42.5{ }^{\circ} \mathrm{C}$ for $30 \mathrm{~min}$. Following heat-shock in the transfected B16 cells, 
the expression of Hsp70 protein was $\sim 60 \%$ compared to $<1 \%$ of the transfected cells without the heat-shock [22]. Moreover, heat-shock has led to an increase in the penetration of Hsp27 and HPV16 E7 DNA constructs, and further expression of proteins in the mesenchymal stem cells. The transfection efficiencies of pEGFP-E7, pEGFP-Hsp27 and pEGFP-E7 + pEGFP-Hsp27 have been reported to be $\sim 26.5, \sim 34$ and $\sim 40.2 \%$, respectively, using Lipofectamine 2000. However, after heating at $42^{\circ} \mathrm{C}$ for 2 hours, these efficiencies were increased to $\sim 51.8, \sim 60.5$ and $\sim 77.5 \%$, respectively [23]. To investigate the effects of Hsp27 on collagen expression during TGF- $\beta 1$-induced differentiation, the Hsp27 plasmid DNA has been transfected using various Lipofectamine 2000 reagent/DNA ratios in A549 cells in which 5 reagent/DNA ratios have been assessed, namely 20:8, 25:8, 30:8, 20:9 and 20:10. The flow cytometry data then have revealed that the transfection efficacies were approximately $83,76,76,70,74 \%$, respectively. These results have indicated that the 20:8 ratio was the optimal liposome to plasmid ratio for using in their next experiments [24].

In current study, the percentage of Hsp27, Hsp27-Nef, Hap70, Hsp70-Nef and Hsp70 + Nef expression in HEK-293T at 2:1 ratio (Lipofectamine 2000 reagent/DNA) was $64.22 \pm$ $0.8,57.1 \pm 0.7,68.8 \pm 1.0,61.7 \pm 0.7$ and $77.4 \pm 1.5 \%$, respectively. Herein, the potency of Hsp70 was more than Hsp27 for increasing Nef-GFP expression in HEK-293T cells. On the other hand, the delivery of pEGFP-hsp70 along with pEGFP-nef increased the rate of GFP population in the cells, indicating a high transfection efficiency as an immunization strategy for the future attempts. Generally, various factors including the transfection reagents, the cell types, the degree of confluency, the DNA quality and quantity, and the reagent/DNA ratios are important parameters that can greatly influence the transfection efficiency. In conclusion, our observations showed that Hsp70 could significantly deliver Nef into the cells and increase its expression as compared to Hsp27. Thus, these constructs will be used as an antigen candidate for development of a HIV-1 DNA vaccine in an animal model in near future.

\section{ACKNOWLEDGEMENT}

The authors would like to thank all those who helped us in this study.

\section{CONFLICT OF INTEREST}

The authors report no conflicts of interest.

\section{REFERENCES}

1. Alter G, Barouch D. Immune correlate-guided HIV vaccine design. Cell host \& microbe. 2018;24(1):25-33. doi:10.1016/j.chom.2018.06.012

2. Liang B, Li H, Li L, Omange RW, Hai Y, Luo M. Current advances in HIV vaccine preclinical studies using Macaque models. Vaccine. 2019;37(26):3388-99. doi:10.1016/j.vaccine.2019.04.094

3. Robinson HL. HIV/AIDS Vaccines: 2018. Clinical Pharmacology \& Therapeutics. 2018;104(6):1062-73. doi:10.1002/cpt.1208

4. Seddiki N, Lévy Y. Therapeutic HIV-1 vaccine: time for immunomodulation and combinatorial strategies. Current Opinion in HIV and AIDS. 2018;13(2):119-27. doi:10.1097/COH.0000000000000444
5. Liu S, Wang S, Lu S. Using DNA Immunization to Elicit Monoclonal Antibodies in Mice, Rabbits, and Humans. Human Gene Therapy. 2018;29(9):997-1003. doi:10.1089/hum.2018.077

6. Gary EN, Weiner DB. DNA vaccines: prime time is now. Current Opinion in Immunology. 2020;65:21-7. doi:10.1016/j.coi.2020.01.006

7. Milani A, Bolhassani A, Shahbazi S, Motevalli F, Sadat SM, Soleymani S. Small heat shock protein 27: An effective adjuvant for enhancement of HIV-1 Nef antigen-specific immunity. Immunology Letters. 2017;191:1622. doi:10.1016/j.imlet.2017.09.005

8. Dekaban GA, Dikeakos JD. HIV-I Nef inhibitors: a novel class of HIVspecific immune adjuvants in support of a cure. AIDS Research and Therapy. 2017;14(1):1-4. doi:10.1186/s12981-017-0175-6

9. Bolhassani A, Shahbazi S, Milani A, Nadji SA. Small heat shock proteins B1 and B6: Which one is the most effective adjuvant in therapeutic HPV vaccine? IUBMB life. 2018;70(10):1002-11. doi:10.1002/iub.1892

10. Milani A, Basirnejad M, Shahbazi S, Bolhassani A. HSP roles as biomarkers and antigens in bacterial and viral infections. Journal of Medical Microbiology and Infectious Diseases. 2016;4(1):1-7.

11. Milani A, Basirnejad M, Bolhassani A. Heat-shock proteins in diagnosis and treatment: an overview of different biochemical and immunological functions. Immunotherapy. 2019;11(3):215-39. doi:10.2217/imt-2018-0105 12. Delneste Y, Larochette V, Jeannin P. Immune Properties of HSP70. InHSP70 in Human Diseases and Disorders 2018 (pp. 173-203). Springer, Cham. doi:10.1007/978-3-319-89551-2_9

13. Milani A, Bolhassani A, Heshmati $\bar{M}$. Delivery of HIV-1 Nef linked to heat shock protein 27 using a cationic polymer is more effective than cationic lipid in mammalian cells. Bratislavske lekarske listy. 2017;118(6):334-8. doi:10.4149/BLL 2017064

14. Suschak JJ, Williams JA, Schmaljohn CS. Advancements in DNA vaccine vectors, non-mechanical delivery methods, and molecular adjuvants to increase immunogenicity. Human vaccines \& immunotherapeutics. 2017;13(12):2837-48. doi:10.1080/21645515.2017.1330236

15. Shi B, Xue M, Wang Y, Wang Y, Li D, Zhao X, et al. An improved method for increasing the efficiency of gene transfection and transduction. International journal of physiology, pathophysiology and pharmacology. 2018;10(2):95

16. Zhou J, Wang L. SAG4 DNA and peptide vaccination provides partial protection against $\mathrm{T}$. gondii infection in BALB/c mice. Frontiers in Microbiology. 2017;8:1733. doi:10.3389/fmicb.2017.01733

17. Kadkhodayan S, Jafarzade BS, Sadat SM, Motevalli F, Agi E, Bolhassani A. Combination of cell penetrating peptides and heterologous DNA prime/protein boost strategy enhances immune responses against HIV-1 Nef antigen in BALB/c mouse model. Immunology letters. 2017;188:38-45. doi:10.1016/j.imlet.2017.06.003

18. Rostami B, Irani S, Bolhassani A, Ahangari Cohan R. Expression of the Recombinant Hsp20-Nef Protein for Evaluation of its Immunogenicity against HIV-1 Nef using Indirect ELISA. Pathobiology Research. 2019;22(3):135-40.

19. Wang T, Green LA, Gupta SK, Kim C, Wang L, Almodovar S, et al. Transfer of intracellular HIV Nef to endothelium causes endothelial dysfunction. PLoS One. 2014;9(3):e91063. doi:10.1371/journal.pone.0091063

20. Yamaoka A, Guan X, Takemoto S, Nishikawa M, Takakura Y Development of a novel Hsp70-based DNA vaccine as a multifunctional antigen delivery system. Journal of controlled release. 2010;142(3):411-5. doi:10.1016/j.jconrel.2009.11.005

21. Zong J, Wang C, Wang Q, Peng Q, Xu Y, Xie X, Xu X. HSP70 and modified HPV 16 E7 fusion gene without the addition of a signal peptide gene sequence as a candidate therapeutic tumor vaccine. Oncology reports. 2013;30(6):3020-6. doi:10.3892/or.2013.2742

22. Andersson HA, Kim YS, O'Neill BE, Shi ZZ, Serda RE. HSP70 promoter-driven activation of gene expression for immunotherapy using gold nanorods and near infrared light. Vaccines. 2014;2(2):216-27. doi:10.3390/vaccines2020216

23. Bolhassani A, Shahbazi S, Agi E, Haghighipour N, Hadi A, Asgari F. Modified DCs and MSCs with HPV E7 antigen and small Hsps: Which one is the most potent strategy for eradication of tumors? Molecular immunology. 2019;108:102-10. doi:10.1016/j.molimm.2019.02.016

24. Deng H, Gao X, Peng H, Wang J, Hou X, Xu H, Yang F. Effect of liposome mediated HSP27 transfection on collagen synthesis in alveolar type II epithelial cells. Molecular medicine reports. 2018;17(5):7319-24. doi:10.3892/mmr.2018.8744 